\title{
Complexity growth in minimal massive 3D gravity
}

\author{
Mohammad M. Qaemmaqami* \\ School of Particles and Accelerators Institute for Research in Fundamental Sciences (IPM) \\ P.O. Box 19395-5531 Tehran, Iran
}

(Received 4 November 2017; published 22 January 2018)

\begin{abstract}
We study the complexity growth by using "complexity = action" (CA) proposal in the minimal massive 3D gravity (MMG) model which is proposed for resolving the bulk-boundary clash problem of topologically massive gravity (TMG). We observe that the rate of the complexity growth for Banados-Teitelboim-Zanelli (BTZ) black hole saturates the proposed bound by physical mass of the BTZ black hole in the MMG model, when the angular momentum parameter and the inner horizon of black hole goes to zero.
\end{abstract}

DOI: $10.1103 /$ PhysRevD.97.026006

\section{INTRODUCTION}

One of the holographic conjectures about the inside of black hole is that its growth is dual to the growth of quantum complexity $[1,2]$. Complexity of a quantum state is defined by the minimum number of simple gates which are needed to build a quantum circuit that constructs them from a reference state.

In the context of AdS/CFT duality, One conjecture is "complexity = volume" $(\mathrm{CV})$, the conjecture is an example of the proposed connection between the tensor network and the geometry of space-time [3-5]. The volume of a maximal spacelike slice into the black hole interior, is proportional to the computational complexity of the dual conformal field theory state [6],

$$
\text { Complexity } \sim \frac{V}{G_{N} l_{\mathrm{AdS}}},
$$

where $V$ is the volume of the Einstein-Rosen bridge (ERB), $l_{\text {AdS }}$ is AdS radius, and $G_{N}$ is Newton's gravitational constant.

The other conjecture is "complexity = action" (CA), the quantum computational complexity of a holographic state is given by the on-shell action on the "Wheeler De-Witt" patch $[2,7]$,

$$
\mathcal{C}(\Sigma)=\frac{S_{W D W}}{\pi \hbar},
$$

where $\Sigma$ is the time slice which is the intersection of any Cauchy surface in the bulk and the asymptotic boundary. It

\footnotetext{
*m.qaemmaqami@ipm.ir
}

Published by the American Physical Society under the terms of the Creative Commons Attribution 4.0 International license. Further distribution of this work must maintain attribution to the author(s) and the published article's title, journal citation, and DOI. Funded by SCOAP ${ }^{3}$. is conjectured that there is an upper bound on the rate of the complexity growth [7]

$$
\frac{d \mathcal{C}}{d t} \leq \frac{2 M}{\pi \hbar}
$$

where $M$ is the mass of black hole. For uncharged black hole the bound is saturated. The above bound is equal to Lloyd's bound which its origin is in quantum computation, if we consider the mass of black hole as the energy of the system. The Aharonov-Anandan-Bohm bound involves the standard deviation of the energy $[8,9]$

$$
\text { orthogonality time } \geq \frac{\pi \hbar}{2 \Delta E},
$$

note that the above inequality is similar to Heisenberg's uncertainty of time-energy principle. The above bound is saturated by a two-state quantum system

$|\psi(t)\rangle=\frac{1}{\sqrt{2}}\left(|0\rangle+e^{i E t}|E\rangle\right) \rightarrow \mid\langle\psi(t) \mid \psi(0)\rangle=\cos \left(\frac{E t}{2 \hbar}\right)$.

To generalize and make precise Lloyd's notion of "operations per second," in [7] authors considered how complexity builds up in an isolated unitary evolving quantum system in a general quantum state. They proposed a similar bound on the complexity growth rate based on the works of Aharonov-Anandan-Bohm, Margolus-Levitin and Lloyd [8-13]. Informally this is the growth rate of the number of simple gates which are needed to construct the state of the computer from a reference state,

$$
\frac{d(\text { number of gates })}{d t} \leq \frac{2 E}{\pi \hbar} .
$$

In this paper we study the complexity growth by using "complexity = action" (CA) conjecture in minimal massive 3D gravity (MMG) model [14], which is proposed for 
resolving the bulk-boundary clash problem of topologically massive gravity (TMG) [15], in some regions of parameter space of the model. The method is adding a new consistent term to the action of the TMG model in the vielbein formalism.

In Sec. II we review shortly the MMG model. In Sec. III we consider the BTZ black hole in MMG model, we introduce the components of vielbein and calculate the nonvanishing components of spin-connection and the auxiliary field of this space-time by solving the equations of motion of MMG model in the vielbein formalism, by plugging the rotating BTZ black hole ansatz. In Sec. IV we consider the well-defined variational principle for MMG model and we find the Gibbons-Hawking term for BTZ black hole solution case.

In Sec. V we calculate the rate of complexity growth by using the "complexity = action" (CA) conjecture in the MMG model and we observe that the rate of the complexity growth for BTZ black hole saturates the proposed bound by physical mass of the BTZ black hole in the MMG model, when the angular momentum parameter and the inner horizon of black hole goes to zero. Finally we consider the TMG limit of the model and we observe that the rate of the complexity growth saturates the proposed bound by physical mass of the BTZ black hole in the TMG model, when the inner horizon of black hole goes to zero. Section VI is devoted to summary.

\section{MINIMAL MASSIVE 3D GRAVITY}

The minimal massive 3D gravity (MMG) [14] is a proposed model for resolving the bulk-boundary clash problem in the topologically massive gravity (TMG)[15], in some region of parameter space of the model. The method is adding a new consistent term to the action of TMG in the vielbein formalism [14]. Note that the bulk-boundary clash problem means that; in TMG we have not positive energy of graviton and unitary dual 2D conformal field theory or positive central charges on the boundary at the same time $[14,16]$. The Lagrangian 3-form of the minimal massive 3D gravity in the vielbein formalism is [14]

$$
\begin{aligned}
L_{\mathrm{MMG}}= & -\sigma e . R+\frac{\Lambda_{0}}{6} e . e \times e+h . T(\omega) \\
& +\frac{1}{2 \mu}\left(\omega . d \omega+\frac{1}{3} \omega . \omega \times \omega\right)+\frac{\alpha}{2} e . h \times h,
\end{aligned}
$$

where $e$, is the vielbein, $\omega$ is the spin-connection and $h$ is the Lagrange multiplier or the auxiliary field. Note that the dot and the cross mean the internal and the external product respectively, the dot implies contraction of the Lorenz indices of two fields with each other and the cross means contraction of the Lorenz indices of two fields with two indices of the Levi-Civita tensor. And also it is worth noting that the linearized equations of motion of MMG is similar with the linearized equations of motion of TMG by making use a redefinition of topological mass parameter [17-19]. Therefor the model has a single local degree of freedom that is realized as a massive graviton in linearization which is similar to TMG.

Note that the action of MMG is only in the vielbein formalism. Because for finding the action in the metric formalism we have to use the equation of motion which is obtained by varying the action with respect to the vielbein field. Therefore we must use the dynamics of the metric for obtaining the action in the metric formalism. Clearly it is not true, because we have to vary the action to find the equations of motion in the metric formalism. Therefore there is the action of MMG only in the vielbein formalism. Then we have to work in the vielbein formalism for calculations in the context of action.

The equations of motion derived from the action of MMG Eq. (2.1) are [14]

$T(\omega)-\alpha e \times h=0, \quad R(\omega)+\mu e \times h+\sigma \mu T(\omega)=0$,

and

$$
-\sigma R(\omega)+\frac{\Lambda_{0}}{2} e \times e+D(\omega) h+\frac{\alpha}{2} h \times h=0 .
$$

The curvature and the torsion 2-forms are defined as follows:

$R(\omega)=d \omega+\frac{1}{2} \omega \times \omega, \quad T(\omega)=d e+\omega \times e$.

To proceed we note that although for generic $\alpha \neq 0$ the torsion, $T(\omega)$ is nonzero, one may define a new torsion free spin-connection, $\Omega=\omega+\alpha h$ by which the Lagrangian 3form reads [19]:

$$
\begin{aligned}
L_{\mathrm{MMG}}(e, \Omega, h)= & -\sigma e \cdot R(\Omega)+\frac{\Lambda_{0}}{6} e \cdot e \times e+h \cdot T(\Omega) \\
& +\frac{1}{2 \mu}\left(\Omega \cdot d \Omega+\frac{1}{3} \Omega \cdot \Omega \times \Omega\right) \\
& -\frac{1}{2 \mu}\left(2 \alpha h \cdot R(\Omega)-\alpha^{2} h \cdot D h+\frac{\alpha^{3}}{3} h \cdot h \times h\right) \\
& +\sigma \alpha e \cdot D h-\frac{\alpha}{2}(1+\sigma \alpha) e \cdot h \times h .
\end{aligned}
$$

In this notation, assuming to have a well-defined variation principle, by varying the above action with respect to the three fields, $(e, \Omega, h)$ we obtain the corresponding equations of motion as follows,

$$
\begin{array}{r}
T(\Omega)=0, \quad R(\Omega)+\frac{\alpha \Lambda_{0}}{2} e \times e+\mu(1+\sigma \alpha)^{2} e \times h=0, \\
D(\Omega) h-\frac{\alpha}{2} h \times h+\sigma \mu(1+\sigma \alpha) e \times h+\frac{\Lambda_{0}}{2} e \times e=0 .
\end{array}
$$

Here the covariant derivative is defined by $D(\Omega) A=$ $d A+\Omega \times A$, where the spin-connection, $\Omega$ is obtained 
from first equation of motion, $T(\Omega)=d e+\Omega \times e=0$, or in other words the torsion-free condition.

\section{BTZ BLACK HOLE}

The metric of the rotating BTZ black hole is given by [20],

$$
\begin{aligned}
d s^{2}= & -\frac{\left(r^{2}-r_{+}^{2}\right)\left(r^{2}-r_{-}^{2}\right)}{l^{2} r^{2}} d t^{2}+\frac{l^{2} r^{2}}{\left(r^{2}-r_{+}^{2}\right)\left(r^{2}-r_{-}^{2}\right)} d r^{2} \\
& +r^{2}\left(d \varphi-\frac{r_{+} r_{-}}{l r^{2}} d t\right)^{2}
\end{aligned}
$$

The mass and the angular momentum parameter of the BTZ black hole, in terms of inner and outer horizons of the black hole and AdS radius, respectively are

$$
M=\frac{r_{+}^{2}+r_{-}^{2}}{8 G l^{2}}, \quad J=\frac{r_{+} r_{-}}{4 G l} .
$$

The nonvanishing components of the vielbein of the rotating BTZ black hole are

$$
\begin{array}{ll}
e_{t}^{0}=\frac{\sqrt{\left(r^{2}-r_{+}^{2}\right)\left(r^{2}-r_{-}^{2}\right)}}{l r}, & e_{t}^{2}=-\frac{r_{+} r_{-}}{l r}, \\
e_{r}{ }^{1}=\frac{l r}{\sqrt{\left(r^{2}-r_{+}^{2}\right)\left(r^{2}-r_{-}^{2}\right)}}, & e_{\varphi}^{2}=r .
\end{array}
$$

Note that the relation between the metric and the vielbein field is $g_{\mu \nu}=\eta_{a b} e_{\mu}{ }^{a} e_{\nu}{ }^{b}$, where $\eta_{a b}$, is the local Minkowski metric.

By the first equation of motion, Eq. (2.6) which is the torsion free condition, one can find the nonvanishing components of the spin-connection from the vielbein field Eq. (3.3) as follows,

$$
\begin{aligned}
\Omega_{t}{ }^{2} & =-\frac{r}{l}, \quad \Omega_{r}{ }^{1}=-\frac{r_{+} r_{-}}{r \sqrt{\left(r^{2}-r_{+}^{2}\right)\left(r^{2}-r_{-}^{2}\right)}}, \\
\Omega_{\varphi}{ }^{0} & =-\frac{\sqrt{\left(r^{2}-r_{+}^{2}\right)\left(r^{2}-r_{-}^{2}\right)}}{r l}, \quad \Omega_{\varphi}{ }^{2}=\frac{r_{+} r_{-}}{l r} .
\end{aligned}
$$

In following we can find the auxiliary field " $h_{\mu}{ }^{a}$," from the second equation of motion, Eq. (2.6), we can rewrite the equation in this form:

$$
R(\Omega)+e \times h^{\prime}=0, \quad h^{\prime}=\frac{\alpha \Lambda_{0}}{2} e+\mu(1+\sigma \alpha)^{2} h,
$$

from the above equation we can find the " $h_{\mu}^{\prime a}$ " [21],

$$
h_{\mu}^{\prime a}=-\operatorname{det}(e)^{-1} \epsilon^{\lambda \rho \sigma}\left(e_{\lambda}^{a} e_{\mu b}-\frac{1}{2} e_{\mu}^{a} e_{\lambda b}\right) R_{\rho \sigma}{ }^{b},
$$

therefore one can find auxiliary field " $h_{\mu}{ }^{a}$ " as follows

$$
\begin{aligned}
h_{\mu}{ }^{a}= & -\frac{1}{\mu(1+\sigma \alpha)^{2}}\left[\operatorname{det}(e)^{-1} \epsilon^{\lambda \rho \sigma}\left(e_{\lambda}{ }^{a} e_{\mu b}-\frac{1}{2} e_{\mu}{ }^{a} e_{\lambda b}\right) R_{\rho \sigma}{ }^{b}\right. \\
& \left.+\frac{\alpha \Lambda_{0}}{2} e_{\mu}{ }^{a}\right] .
\end{aligned}
$$

We find the non vanishing components of $h_{\mu}{ }^{a}$, by replacing the vielbein and spin-connection fields in the above relation Eq. (3.7), as follows:

$$
\begin{aligned}
h_{t}^{0} & =\frac{1-\alpha l^{2} \Lambda_{0}}{2 \mu l^{2}(1+\sigma \alpha)^{2}} \frac{\sqrt{\left(r^{2}-r_{+}^{2}\right)\left(r^{2}-r_{-}^{2}\right)}}{l r}, \\
h_{t}{ }^{2} & =-\frac{1-\alpha l^{2} \Lambda_{0}}{2 \mu l^{2}(1+\sigma \alpha)^{2}} \frac{r_{+} r_{-}}{l r}, \\
h_{r}{ }^{1} & =\frac{1-\alpha l^{2} \Lambda_{0}}{2 \mu l^{2}(1+\sigma \alpha)^{2}} \frac{l r}{\sqrt{\left(r^{2}-r_{+}^{2}\right)\left(r^{2}-r_{-}^{2}\right)}}, \\
h_{\varphi}{ }^{2} & =\frac{1-\alpha l^{2} \Lambda_{0}}{2 \mu l^{2}(1+\sigma \alpha)^{2}} r .
\end{aligned}
$$

One can see easily for the BTZ black hole we have,

$$
h_{\mu}{ }^{a}=\left(\frac{1-\alpha l^{2} \Lambda_{0}}{2 \mu l^{2}(1+\sigma \alpha)^{2}}\right) e_{\mu}{ }^{a} .
$$

We can use this relation for finding the Gibbons-Hawking term in the BTZ black hole case, by a well-defined variational principle in the Dirichlet boundary condition.

\section{VARIATIONAL PRINCIPLE}

The equations of motion, Eq. (2.6) rely on the fact that the model admits a well-imposed variational principle. This procedure requires the proper Gibbons-Hawking term to make sure that all boundary terms can be consistently removed. In this section we would like to reexamine the well-defined variation of the action leading to the corresponding equations of motion.

To proceed let us consider the action of the MMG model Eq (2.5) whose variation with respect to the fields $e, \Omega$, and $h$ are given by [19];

$$
\begin{aligned}
\delta_{e} L(e, \Omega, h) & =E_{e} \cdot \delta e-D(\Omega)(h \cdot \delta e), \\
\delta_{\Omega} L(e, \Omega, h)= & E_{\Omega} \cdot \delta \Omega \\
& +D(\Omega)\left(\sigma e \cdot \delta \Omega-\frac{1}{2 \mu}(\Omega \cdot \delta \Omega-2 \alpha h \cdot \delta \Omega)\right), \\
\delta_{h} L(e, \Omega, h)= & E_{h} \cdot \delta h \\
& +D(\Omega)\left(-\sigma \alpha e \cdot \delta h-\frac{1}{2 \mu}\left(\alpha^{2} h \cdot \delta h-2 \alpha \Omega \cdot \delta h\right)\right) .
\end{aligned}
$$

Using the Stokes' theorem the corresponding boundary terms appearing in the above variation may be recast to the following form 


$$
\begin{aligned}
\left.\delta S\right|_{\text {boundary }} & =\frac{1}{8 \pi G} \int_{\partial \mathcal{M}} d^{2} x \epsilon^{i j}\left(h_{i a} \delta e_{j}^{a}+\left[\sigma e-\frac{1}{2 \mu} \Omega+\frac{\alpha}{\mu} h\right]_{i a} \delta \Omega_{j}^{a}\right. \\
& \left.+\left[-\alpha \sigma e+\frac{\alpha}{\mu} \Omega-\frac{\alpha^{2}}{2 \mu} h\right]_{i a} \delta h_{j}^{a}\right) .
\end{aligned}
$$

For a well-defined variational principle, we need a Gibbons-Hawking term to cancel the boundary terms in the Dirichlet boundary condition. In general finding the Gibbons-Hawking term is difficult for this model, but in the case of BTZ black hole solution, the " $h_{\mu}{ }^{a}$ " field is proportional with the vielbein field, " $e_{\mu}{ }^{a}$ ", Eq. (3.9) therefore the variation of the auxiliary field " $h_{\mu}{ }^{a}$ " is as follows:

$$
\delta h_{\mu}{ }^{a}=\left(\frac{1-\alpha l^{2} \Lambda_{0}}{2 \mu l^{2}(1+\sigma \alpha)^{2}}\right) \delta e_{\mu}{ }^{a},
$$

then the third term of variation of action on the boundary Eq. (4.2) is canceled by the Dirichlet boundary condition therefore the Gibbons-Hawking term in this case is

$$
\begin{aligned}
S_{\mathrm{GH}} & =-\frac{1}{8 \pi G} \int_{\partial \mathcal{M}} d^{2} x \epsilon^{i j}\left(\sigma \tilde{e}+\frac{\alpha}{\mu} \tilde{h}\right)_{i a} \tilde{\Omega}_{j}^{a} \\
& =-\frac{1}{8 \pi G}\left[\sigma+\alpha\left(\frac{1-\alpha l^{2} \Lambda_{0}}{2 \mu^{2} l^{2}(1+\sigma \alpha)^{2}}\right)\right] \int_{\partial \mathcal{M}} d^{2} x \epsilon^{i j} \tilde{e}_{i a} \tilde{\Omega}_{j}^{a},
\end{aligned}
$$

where $\tilde{e}, \tilde{\Omega}$, and $\tilde{h}$ are the boundary vielbein, the boundary spin-connection, and the boundary auxiliary field, respectively.

\section{COMPUTATION OF COMPLEXITY GROWTH IN MINIMAL MASSIVE 3D GRAVITY}

In the proposal known as "complexity = action" (CA) the quantum computational complexity of a holographic state is given by the on-shell action evaluated on a bulk region known as the "Wheeler-De Witt" patch [2,7]

$$
\mathcal{C}(\Sigma)=\frac{S_{W D W}}{\pi \hbar}
$$

it is conjectured that there is an upper bound on the rate of the complexity growth [7]

$$
\frac{d \mathcal{C}}{d t} \leq \frac{2 M}{\pi \hbar}
$$

where $M$ is the mass of black hole. For uncharged black hole the bound is saturated. Note that here we use the complexity $=$ action $(\mathrm{CA})$ conjecture, because the bound on complexity growth rate in complexity $=$ action $(\mathrm{CA})$ is exactly the Lloyd's bound. And also it is worth noting that, however the first proposal was complexity $=$ volume $(\mathrm{CV})$, but community have paid more attention to the complexity $=$ action $(\mathrm{CA})$ conjecture and people have done more work in this context.

The corresponding action of minimal massive 3D gravity containing the Gibbons-Hawking term is given by

$$
\begin{aligned}
S= & S_{\mathcal{M}}+S_{\partial \mathcal{M}} \\
= & \frac{1}{16 \pi G} \int_{\mathcal{M}} d^{3} x \epsilon^{\lambda \mu \nu}\left[L_{\mathrm{MMG}}(e, \Omega, h)\right]_{\lambda \mu \nu} \\
& -\frac{1}{8 \pi G}\left[\sigma+\alpha\left(\frac{1-\alpha l^{2} \Lambda_{0}}{2 \mu^{2} l^{2}(1+\sigma \alpha)^{2}}\right)\right] \int_{\partial \mathcal{M}} d^{2} x \epsilon^{i j} \tilde{e}_{i a} \tilde{\Omega}_{j}^{a},
\end{aligned}
$$

where $\left[L_{\mathrm{MMG}}(e, \Omega, h)\right]_{\lambda \mu \nu}$ is the Lagrangian 3-form of MMG which is defined in Sec. II by Eq. (2.5).

To compute the rate of the complexity growth, we should calculate the difference between the on-shell actions which are evaluated over two nearby WDW patches [22]. In the present case by BTZ solution at the late time, only the region between the inner and outer horizons contributes to this difference. Therefore we find;

$$
\begin{aligned}
\delta S_{\mathcal{M}} & =S_{\mathcal{M}}\left[\left.W D W\right|_{t+\delta t}\right]-S_{\mathcal{M}}\left[\left.W D W\right|_{t}\right]=\frac{1}{16 \pi G} \int_{t}^{t+\delta t} \int_{r_{-}}^{r^{+}} \int_{0}^{2 \pi} \epsilon^{\lambda \mu \nu}\left[L_{\mathrm{MMG}}(e, \Omega, h)\right]_{\lambda \mu \nu} d t d r d \varphi \\
& =-\frac{2 \pi \delta t}{16 \pi G}\left[\sigma+\alpha\left(\frac{1-\alpha l^{2} \Lambda_{0}}{2 \mu^{2} l^{2}(1+\sigma \alpha)^{2}}\right)\right] \int_{r_{-}}^{r^{+}} \frac{4 r}{l^{2}}=-\left[\sigma+\alpha\left(\frac{1-\alpha l^{2} \Lambda_{0}}{2 \mu^{2} l^{2}(1+\sigma \alpha)^{2}}\right)\right]\left(\frac{r_{+}^{2}-r_{-}^{2}}{4 G l^{2}}\right) \delta t .
\end{aligned}
$$

In following the contribution of the Gibbons-Hawking term Eq. (4.4) is given by

$$
\begin{aligned}
\delta S_{\partial \mathcal{M}} & =-\frac{1}{8 \pi G}\left[\sigma+\alpha\left(\frac{1-\alpha l^{2} \Lambda_{0}}{2 \mu^{2} l^{2}(1+\sigma \alpha)^{2}}\right)\right] \int_{\partial \mathcal{M}} d^{2} x \epsilon^{i j} \tilde{e}_{i a} \tilde{\Omega}_{j}^{a} \\
& =\frac{1}{8 \pi G}\left[\sigma+\alpha\left(\frac{1-\alpha l^{2} \Lambda_{0}}{2 \mu^{2} l^{2}(1+\sigma \alpha)^{2}}\right)\right]\left(\left.\int_{t}^{t+\delta t} \int_{0}^{2 \pi} \frac{1}{l^{2}}\left(2 r^{2}-r_{+}^{2}-r_{-}^{2}\right) d t d \varphi\right|_{r_{+}}-\left.\int_{t}^{t+\delta t} \int_{0}^{2 \pi} \frac{1}{l^{2}}\left(2 r^{2}-r_{+}^{2}-r_{-}^{2}\right) d t d \varphi\right|_{r_{-}}\right) \\
& =\left[\sigma+\alpha\left(\frac{1-\alpha l^{2} \Lambda_{0}}{2 \mu^{2} l^{2}(1+\sigma \alpha)^{2}}\right)\right]\left(\frac{r_{+}^{2}-r_{-}^{2}}{2 G l^{2}}\right) \delta t
\end{aligned}
$$


Therefore, we find the rate of the complexity growth by Eq. (5.1)

$$
\begin{aligned}
\dot{\mathcal{C}} & =\frac{1}{\pi \hbar} \frac{d S}{d t} \equiv \frac{1}{\pi \hbar}\left(\frac{d S_{\mathcal{M}}}{d t}+\frac{d S_{\partial \mathcal{M}}}{d t}\right) \\
& =\frac{1}{\pi \hbar}\left[\sigma+\alpha\left(\frac{1-\alpha l^{2} \Lambda_{0}}{2 \mu^{2} l^{2}(1+\sigma \alpha)^{2}}\right)\right] \frac{r_{+}^{2}-r_{-}^{2}}{4 G l^{2}} .
\end{aligned}
$$

We know the mass of BTZ black hole in MMG theory [23-25]

$\mathcal{M}_{\mathrm{MMG}}=\left[\sigma+\alpha\left(\frac{1-\alpha l^{2} \Lambda_{0}}{2 \mu^{2} l^{2}(1+\sigma \alpha)^{2}}\right)\right]\left(\frac{r_{+}^{2}+r_{-}^{2}}{8 G l^{2}}\right)+\frac{r_{+} r_{-}}{4 G \mu l^{3}}$,

one can see for $r_{-}=0$, which is the nonrotating BTZ black hole case in 3D Einstein Gravity (the angular momentum parameter is zero), we have

$$
\dot{\mathcal{C}}=\frac{1}{\pi \hbar} \frac{d S}{d t}=\frac{2 \mathcal{M}_{\mathrm{MMG}}}{\pi \hbar},
$$

it is interesting, one can see the rate of the complexity growth saturates the proposed bound [7] by the physical mass of the BTZ black hole in the minimal massive 3D gravity model.

Finally let us consider the TMG limit of the model when $\alpha=0$,

$\dot{\mathcal{C}}=\frac{1}{\pi \hbar} \frac{d S}{d t} \equiv \frac{1}{\pi \hbar}\left(\frac{d S_{\mathcal{M}}}{d t}+\frac{d S_{\partial \mathcal{M}}}{d t}\right)=\frac{\sigma}{\pi \hbar}\left(\frac{r_{+}^{2}-r_{-}^{2}}{4 G l^{2}}\right)$.

We know the mass of BTZ black hole in TMG theory $[23,26]$,

$$
\mathcal{M}_{\mathrm{TMG}}=\sigma\left(\frac{r_{+}^{2}+r_{-}^{2}}{8 G l^{2}}\right)+\frac{r_{+} r_{-}}{4 G \mu l^{3}},
$$

one can see for $r_{-}=0$, we have

$$
\dot{\mathcal{C}}=\frac{1}{\pi \hbar} \frac{d S}{d t}=\frac{2 \mathcal{M}_{\mathrm{TMG}}}{\pi \hbar},
$$

clearly the rate of the complexity growth saturates the proposed bound [7] by the physical mass of the BTZ black hole in the topologically massive gravity model.

\section{SUMMARY}

In this work we study the complexity growth by using the complexity = action (CA) conjecture in minimal massive 3D gravity (MMG) model which is proposed for resolving the bulk-boundary clash problem of topologically massive gravity (TMG). We observe that the rate of the complexity growth for BTZ black hole saturates the proposed bound by physical mass of the BTZ black hole in the MMG model, when the angular momentum parameter and the inner horizon of black hole goes to zero. We can say it is another evidence for the hypothesis that black holes are the fastest computers in Nature [2], as they are the fastest scramblers [27].

Recently some work have been done on quantum complexity in context of holography and black holes [22,28-45], and also it needs more investigations and calculations in the other Gravity models as higher derivative Gravity models and more black hole solutions, in addition it seems interesting to study the connection between holographic complexity and tensor networks or butterfly effect in different context and models to achieve deeper understanding of these important and interesting phenomenon of the nature.

\section{ACKNOWLEDGMENTS}

I am grateful to thank Ali Naseh for useful discussions. I also thank the referee for her/his useful comments. I would like to thank the Institute for Research in Fundamental Sciences (IPM) and the Science Ministry of IRAN for research facilities and financial support.

Note added.-As this work was being completed, [44] appeared which has minor overlap with the present paper.
[1] L. Susskind, Computational complexity and black hole horizons, Fortschr. Phys. 64, 24 (2016); Addendum to computational complexity and black hole horizons, Fortschr. Phys. 64, 44 (2016).

[2] A. R. Brown, D. A. Roberts, L. Susskind, B. Swingle, and Y. Zhao, Holographic Complexity Equals Bulk Action?, Phys. Rev. Lett. 116, 191301 (2016).

[3] B. Swingle, Entanglement renormalization and holography, Phys. Rev. D 86, 065007 (2012).

[4] G. Vidal, Entanglement Renormalization, Phys. Rev. Lett. 99, 220405 (2007).
[5] T. Hartman and J. Maldacena, Time evolution of entanglement entropy from black hole interiors, J. High Energy Phys. 05 (2013) 014.

[6] D. Stanford and L. Susskind, Complexity and shock wave geometries, Phys. Rev. D 90, 126007 (2014).

[7] A. R. Brown, D. A. Roberts, L. Susskind, B. Swingle, and Y. Zhao, Complexity, action, and black holes, Phys. Rev. D 93, 086006 (2016).

[8] J. Anandan and Y. Aharonov, Geometry of Quantum Evolution, Phys. Rev. Lett. 65, 1697 (1990). 
[9] Y. Aharonov and D. Bohm, Time in the quantum theory and the uncertainty relation for time and energy, Phys. Rev. 122, 1649 (1961).

[10] N. Margolus and L. B. Levitin, The maximum speed of dynamical evolution, Physica D (Amsterdam) 120, 188 (1998).

[11] S. Lloyd, Computational Capacity of the Universe, Phys. Rev. Lett. 88, 237901 (2002).

[12] S. Lloyd, The computational universe: Quantum gravity from quantum computation, arXiv:quant-ph/0501135.

[13] S. Lloyd, Quantum limits to the measurement of spacetime geometry, arXiv:quant-ph/0505064.

[14] E. Bergshoeff, O. Hohm, W. Merbis, A. J. Routh, and P. K. Townsend, Minimal massive 3D gravity, Classical Quantum Gravity 31, 145008 (2014).

[15] S. Deser, R. Jackiw, and S. Templeton, Topologically massive gauge theories, Ann. Phys. (N.Y.) 140, 372 (1982); Topologically massive gauge theories, Ann. Phys. (N.Y.) 281, 409 (2000); Erratum, Ann. Phys. (N.Y.) 185, 406(E) (1988).

[16] G. Alkac, L. Basanisi, E. Kilicarslan, and B. Tekin, Unitarity problems in $3 D$ gravity theories, Phys. Rev. D 96, 024010 (2017).

[17] B. Tekin, Minimal massive gravity: Conserved charges, excitations and the chiral gravity limit, Phys. Rev. D 90, 081701 (2014).

[18] M. Alishahiha, M. M. Qaemmaqami, A. Naseh, and A. Shirzad, On 3D minimal massive gravity, J. High Energy Phys. 12 (2014) 033.

[19] M. Alishahiha, M. M. Qaemmaqami, A. Naseh, and A. Shirzad, Holographic renormalization of 3D minimal massive gravity, J. High Energy Phys. 01 (2016) 106.

[20] M. Banados, C. Teitelboim, and J. Zanelli, The Black Hole in Three-Dimensional Space-time, Phys. Rev. Lett. 69, 1849 (1992).

[21] W. Merbis, Chern-Simons-like Theories of Gravity, arXiv:1411.6888.

[22] M. Alishahiha, A. Faraji Astaneh, A. Naseh, and M. H. Vahidinia, On complexity for $\mathrm{F}(\mathrm{R})$ and critical gravity, J. High Energy Phys. 05 (2017) 009.

[23] S. Nam and J. D. Park, Mass and angular momentum of black holes in 3D gravity theories with first order formalism and new type black hole in minimal massive gravity, arXiv:1606.05997.

[24] M. R. Setare and H. Adami, Black hole conserved charges in generalized minimal massive gravity, Phys. Lett. B 744, 280 (2015).

[25] D. Mahdavian Yekta, Hamiltonian formalism of minimal massive gravity, Phys. Rev. D 92, 064044 (2015).

[26] A. Bouchareb and G. Clement, Black hole mass and angular momentum in topologically massive gravity, Classical Quantum Gravity 24, 5581 (2007).
[27] Y. Sekino and L. Susskind, Fast scramblers, J. High Energy Phys. 10 (2008) 065.

[28] M. Alishahiha, Holographic complexity, Phys. Rev. D 92 , 126009 (2015).

[29] S. Chapman, H. Marrochio, and R. C. Myers, Complexity of formation in holography, J. High Energy Phys. 01 (2017) 062.

[30] R. G. Cai, S. M. Ruan, S. J. Wang, R. Q. Yang, and R. H. Peng, Action growth for AdS black holes, J. High Energy Phys. 09 (2016) 161.

[31] W. J. Pan and Y.C. Huang, Holographic complexity and action growth in massive gravities, Phys. Rev. D 95, 126013 (2017).

[32] R. Q. Yang, Strong energy condition and complexity growth bound in holography, Phys. Rev. D 95, 086017 (2017).

[33] D. Carmi, R. C. Myers, and P. Rath, Comments on holographic complexity, J. High Energy Phys. 03 (2017) 118.

[34] R. Q. Yang, C. Niu, and K. Y. Kim, Surface counterterms and regularized holographic complexity, J. High Energy Phys. 09 (2017) 042.

[35] R. G. Cai, M. Sasaki, and S. J. Wang, Action growth of charged black holes with a single horizon, Phys. Rev. D 95, 124002 (2017).

[36] E. Bakhshaei, A. Mollabashi, and A. Shirzad, Holographic subregion complexity for singular Surfaces, Eur. Phys. J. C 77, 665 (2017).

[37] F. J. G. Abad, M. Kulaxizi, and A. Parnachev, On complexity of holographic flavors, arXiv:1705.08424.

[38] A. Reynolds and S. F. Ross, Complexity in de sitter space, Classical Quantum Gravity 34, 175013 (2017).

[39] J. Tao, P. Wang, and H. Yang, Testing holographic conjectures of complexity with Born-Infeld black holes, Eur. Phys. J. C 77, 817 (2017).

[40] W. D. Guo, S. W. Wei, Y. Y. Li, and Y. X. Liu, Complexity growth rates for AdS black holes in massive gravity and $f(R)$ gravity, arXiv:1703.10468.

[41] M. Alishahiha and A. Faraji Astaneh, Holographic fidelity susceptibility, Phys. Rev. D 96, 086004 (2017).

[42] K. Nagasaki, Complexity of $\mathrm{AdS}_{5}$ black holes with a rotating string, Phys. Rev. D 96, 126018 (2017).

[43] Y. G. Miao and L. Zhao, Complexity/action duality of the shock wave geometry in a massive gravity theory, arXiv:1708.01779 [Phys. Rev. D (to be published)].

[44] M. Ghodrati, On complexity growth in massive gravity theories, the effects of chirality and more, Phys. Rev. D 96, 106020 (2017).

[45] S. A. Hosseini Mansoori and M. M. Qaemmaqami, Complexity growth, butterfly velocity and black hole thermodynamics, arXiv:1711.09749. 